M. Compendium. A Guide to M.'s Life and Music, hrsg. von H.C.R. LANDon, Ldn. 1990; dt. Mn. 1991. KüsteR, K.: M. Eine musikalische Biographie, Stg. 1990; engl. Oxford 1996. Ders.: Formale Aspekte des ersten Allegros in M.s Konzerten, Kassel 1991. KNePLeR, G.: W.A. M. Annäherungen, Bln. 1991. WolfF, CHR.: M.s Requiem, Mn. 1991. M. Studies, hrsg. von Cl. Ersen, 2 Bde., Oxford 1991 und 1997. Ford, C.: Cosi? Sexual Politics in M.s Operas, Manchester 1991. NAGEL, I.: Autonomie und Gnade. Über M.s Opern, Mn. und Kassel 1991, zugleich engl. Cambridge 1991. M. in der Musik des 20. Jahrhunderts. Formen ästhetischer und kompositionstechnischer Rezeption, hrsg. von W. GraTzER und S. Mauser, Laaber 1992. Konrad, U.: M.s Schaffensweise, Göttingen 1992. SchulER, H.: M. und die Freimaurerei, Wilhelmshaven 1992. Intern. Mw. Kgr. zum M.jahr 1991, hrsg. von I. Fuchs, Tutzing 1993. M.s Streichquintette, hrsg. von CL. EISEN und W.-D. SeIffert, Stg. 1994. Heartz, D.: Haydn, M. and the Viennese School, 1740-1780, N.Y. 1995. Powers, H.S.: Reading M.'s Music. Text and Topic, Sense and Syntax in Current Musicology (57) 1995, 5-44. Rampe, S.: M.s Claviermusik. Klangwelt und Aufführungspraxis, Kassel 1995. Taruskin, R.: Text and Act. Essays on Music and Performance, Oxford 1995. Solomon, M.: A Life, N.Y. 1995. W.A. M.: Essays on his Life and Music, hrsg. von St. Sadie, Oxford 1996; dt. Ffm. 1996. M's Piano Concertos, hrsg. von N. ZasLaW, Ann Arbor 1996. WILLASCHEK, W.: M.-Theater. Vom Idomeneo bis zur Zauberflöte, Stg. und Weimar 1996. Opera buffa in M.'s Vienna, hrsg. von M. Hunter und J. Webster, Cambridge 1997. IRving, J.: The Haydn Quartets, Cambridge 1998. Hunter, M.: The Culture of Opera Buffa in M.'s Vienna. A Poetics of Entertainment, Princeton (NJ) 1999. HÜPPE, E.: W.A. M. Innovation und Praxis. Zum Quintett Es-Dur KV 452, Mn. 1998 (MK 99). KüstER, K.: W.A. M. und seine Zeit, Laaber 2001. The Cambridge Companion to M., hrsg. von S.B. KeEFE, Cambridge 2003.

Wolfgang Ruf

\section{Muffat, Georg}

Getauft 1. 6. 1653 in Mégève (Savoyen); gest. 23. 2. 1704 in Passau

Schwerpunkt von M.s Schaffen, das ausschließlich zwischen 1677 und 1695 entstand, war die Instrumentalmusik. Dies mag damit zusammenhängen, daß $M$. zunächst bei Lully, später dann bei Corelli Komposition studiert hatte; beide waren ebenfalls sehr stark - bzw. im Falle Corellis fast ausschließlich - an Instrumentalmusik interessiert. Bei ihnen lernte M. den französischen bzw. den italienischen Stil kennen, die er dann beide mit deutschen und österreichischen Stilelementen verband. In dieser Verbindung zum sogenannten "vermischten Stil« liegt M.s Bedeutung für die Entwicklung der deutschen Instrumentalmusik.

Die 1677 in Prag geschriebene Violinsonate M.s, die als einzige Komposition im Autograph vorliegt, folgt in ihrer Anlage der italienischen Kirchensonate. Ebenfalls italienisch geprägt ist die 1682 erschienene Sonatensammlung Armonico tributo, deren Stücke sowohl in kleiner als auch in großer Besetzung aufführbar sind. In den fünf Stimmheften werden daher auch Solo- und Tuttipassagen gekennzeichnet, so daß bereits Concerti grossi vorliegen. Entsprechend konnte $M$. in seiner 1701 veröffentlichten Auserlesenen Instrumentalmusik diese Sonaten ohne Probleme zu sechs Concerti grossi umarbeiten, sechs weitere komponierte er neu hinzu. Für diese Sammlung nun ließ er acht Stimmen drucken, die sich auf das Concertino und das Concerto grosso verteilen. In der Vorrede macht $M$. deutlich, daß er ganz bewußt eine Verschmelzung italienischer und französischer Elemente angestrebt hat. Mit einer solchen Synthese verschiedener Nationalstile bahnt $M$. den Weg für den "vermischten Stil", wie er später z. B. für Telemann charakteristisch wird. Anders als diese Sammlungen enthalten die beiden Bände Florilegium I und II (1695 und 1698) Orchestersuiten nach französischem Muster, die auch hinsichtlich des Instrumentariums dem Vorbild Lullys folgen. Die Vorreden enthalten wichtige Aussagen zur Aufführungspraxis nach der "Lullianisch-Frantzösischen Arth«. Züge des vermischten Stils zeigt auch M.s Apparatus musico-organisticus (1690), wenngleich vieles an die Toccaten Frescobaldis erinnert, der von $M$. auch als Vorbild ausdrücklich genannt wird. M.s Sohn Gottlieb (1690-1770) trat mit bedeutsamer Musik für Tasteninstrumente hervor.

Noten: Florilegium I und II, hrsg. von H. RIETsCH, Wien (1894 und 1895); Graz 1959 (DTÖ 2 \& 4). Auserlesene Instrumentalmusik u. a., hrsg. von E. LuNTz, Wien 1904 (DTO 23). Armonico tributo u. a., hrsg. von E. SCHENK, Wien 1953 (DTÖ 89). Apparatus musicoorganisticus, hrsg. von R. WALTER, Altötting (1957); ${ }^{2} 1968$.

Dokumente: Regulae concentuum partiturae. An Essay on Thoroughbass, hrsg. von H. Federhofer, NA Rom 1961.

Literatur: Stampfl, I.: G. M.s Orchesterkompositionen, Passau 1984 [mit WV und Bibl.]. Kolneder, W.: G.M. zur Aufführungspraxis, Straßburg $1970 ;{ }^{2} 1990$.

Reinmar Emans 